\title{
Histerossonografia Tridimensional em Infertilidade: Estudo Preliminar
}

\author{
Three-dimensional Hysterosonography in Infertility: Preliminary Study
}

Nilka F. Donadio, Nilson Donadio, Mychelle M. G. Torres

\section{RESUM0}

Objetivo: comparar as informações obtidas pela ultra-sonografia pélvica e transvaginal (USG), histerossalpingografia (HSG), histeroscopia diagnóstica (HSC), ressonância nuclear magnética pélvica (RNMP) e hísterossonografia tridimensional (HSSNG 3D) para melhorar a acurácia e simplificar a investigação do fator uterino cervical e corporal em infertilidade conjugal.

Métodos: no período de janeiro a julho de 2003, cinquenta mulheres com queixa de infertilidade de, no mínimo, dois anos foram submetidas a USG, HSG, HSC, RNMP e HSSNG $3 D$ como exames de rastreamento para o diagnóstico do fator uterino. Foram analisados o canal endocervical, endométrio, miométrio e presença de malformações uterinas. Os resultados de cada exame foram analisados e comparados.

Resultados: das 50 mulheres incluidas, 12 (24\%) apresentaram alteração em pelo menos um dos exames realizados. Quando se comparou a HSSNG 3D com a USG, a HSSNG 3D forneceu informações adicionais em 7 casos (58,3\%); comparada com a HSG, forneceu informações adicionais em 7 casos (58,3\%); com a HSC, em 4 casos (32,1\%), e comparada à RNPM, em 6 casos (50\%). Somente em dois casos a HSG detectou alterações do canal endocervical que não foram visualizadas na HSSNG 3D; em todos os outros casos a HSSNG 3D forneceu o mesmo diagnóstico ou deu informações adicionais em relação aos outros exames. A análise pelo teste kappa mostrou que existe concordância entre os diagnósticos obtidos com o uso da HSSNG 3D e a USG, HSG e RNMP. No entanto, quando se associaram os resultados da HSG e da HSSNG 3D observamos que todas as condições clínicas poderiam ter sido diagnosticadas, de forma precisa, somente com esses exames.

Conclusão: a associação da HSG com a HSSNG 3D pode ser suficiente para o diagnóstico do fator uterino cervical e corporal em infertilidade, reduzindo o número de exames realizados por cada paciente, os custos totais finais, a ansiedade e o retardo para o início do tratamento.

PALAVRAS-CHAVE: Infertilidade. Histerossalpingografia. Ressonância nuclear magnética. Histeroscopia.

\section{Introdução}

O diagnóstico do fator uterino associado à infertilidade compreende avaliação seqüencial dos antecedentes pessoais e familiares e, mais diretamente, do exame ginecológico. As condições anatômicas do colo, canal cervical, istmo e corpo

Clínica de Infertilidade Conjugal do Departamento de Obstetrícia e Ginecologia da Irmandade da Santa Casa de Misericórdia de São Paulo e Pró-Embryo. São Paulo-SP. Correspondência:

Mychelle de Medeiros Garcia Torres

Rua Dr. Nicolau de Sousa Queiros, 406 apto 205 - Vila Mariana 04105-001 - São Paulo - SP

Fone: (11) 5573-0408 - 9383-1648

e-mail: mytorres@ig.com.br uterino são delineadas segundo a história clínica do tempo de infertilidade por meio da pesquisa do ciclo menstrual e outros sintomas relacionados como dismenorréia, dispareunia, sinusiorragia, dor acíclica, perdas sangüíneas atípicas, traumas, afecções, abortamentos repetidos ou provocados, curetagens e outras intervenções ${ }^{1}$.

A pesquisa básica anatomicofuncional deve ser realizada pela ecografia simples transvaginal (USG), que deve ser sempre relacionada à dinâmica folículo-luteal, e da histerossalpingografia (HSG) ${ }^{2}$, que não pode ser descartada enquanto não tivermos faloposcopias ou tuboscopias confiáveis no concernente aos detalhes do endossalpíngeo.

A associação da USG e HSG nos informa so- 
bre as condições do canal cervical, do endométrio, do relevo da cavidade uterina, alterações miometriais, além de permitir a suspeita de outros fatores de infertilidade feminina, gerados por seqüelas de afecções pélvicas altas.

$\mathrm{Na}$ suspeita de anomalia uterina, indica-se complementação desta avaliação inicial por meio da vídeo-histeroscopia, cuja falta de correlações prévias com USG e HSG pode não diagnosticar alterações cervicais, subendometriais e miometriais que pouco modificam a cavidade uterina ${ }^{1}$. Como exemplo, a estenose cervical relativa não diagnosticada pode prejudicar os resultados da fertilização in vitro pela dificuldade durante a transferência de embriões ${ }^{1}$; miomas e adenomiose, possiveis responsáveis por contrações uterinas atípicas, disperistaltismo e spottings, podem comprometer a migração dos gametas além da implantação ${ }^{2,3}$.

A insuficiência da USG isolada para avaliação da cavidade uterina, as falhas da HSG e a discordância desta com as endoscopias em 30\% dos $\operatorname{casos}^{4,5}$, principalmente quanto ao fator uterino, têm acentuado a necessidade de enriquecer essas provas com as informações obtidas pela histerossonografia ${ }^{6}$ e até ressonância nuclear magnética pélvica (RNMP) para estudo adequado dos miomas, adenomiomas e para avaliação da zona de junção.

Uma vez que se deseja diminuir o número total de provas necessárias para um completo diagnóstico do fator uterino, propomos a realização da hístero-sonografia tridimensional (HSSNG 3D) em infertilidade conjugal. O exame seria agendado pelo ginecologista e realizado durante a USG convencional de rotina. A distensão da cavidade com soro fisiológico e aplicação do recurso 3D, oferece imagens da cavidade uterina e do endométrio similares à histeroscopia (HSC), além de fornecer informações sobre o miométrio, incluindo as condições vasculares pela dopplerfluxometria e condições dos ovários ${ }^{7}$. O exame poderia antecipar dificuldades cirúrgicas, possibilitando a escolha adequada da via e tática de abordagem e substituiria, inclusive, a RNMP, atualmente solicitado no pré-operatório com freqüência crescente em certas doenças. Os cortes arquivados pelo aparelho possibilitam boa avaliação global do útero por meio da reconstituição de imagens.

Este trabalho tem como objetivo, portanto, comparar as informações obtidas pela HSSNG 3D com as obtidas pela HSG, USG convencional, HSC diagnóstica e RNMP na avaliação do fator uterino em infertilidade conjugal.

\section{Pacientes e Métodos}

Foram selecionadas na Clínica de Infertilidade Conjugal do Departamento de Obstetrícia e Ginecologia da Irmandade da Santa Casa de Misericórdia de São Paulo e na Clínica Pró-embryo (São Paulo) 50 mulheres com idade entre 26 e 35 anos, com história de casamento infértil há pelo menos 2 anos, sem antecedentes relevantes. A seleção foi feita incluindo no estudo os 25 primeiros casos novos que procuraram as duas Instituições no ano de 2003, no período de fevereiro a julho. O trabalho foi aprovado pela Comissão de Ética em Pesquisa da Instituição da Irmandade da Santa Casa de Misericórdia de São Paulo e todas as pacientes receberam e assinaram termo de consentimento informado.

Foram realizados os exames de rotina da pesquisa básica anatomicofuncional em infertilidade: HSG e USG, além da HSC, RNMP e HSSNG 3D. O estudo compara os achados do USG, HSG, histeroscopia, HSSNG 3D e RNMP. Os exames foram realizados em três ciclos menstruais distintos, para que os exames invasivos não provocassem alterações endometriais que causassem confusão no diagnóstico de outro exame, na seguinte ordem: USG precedendo a HSG, ambas realizadas no mesmo dia; HSC e RNMP realizadas no ciclo seguinte e HSSNG 3D no terceiro ciclo. No presente estudo, temos como objetivo analisar o fator uterino corporal e cervical, não sendo considerados os fatores anatômicos tubo-peritoneais ou alterações ovarianas. As provas foram realizadas por profissionais experientes com laudos sem o conhecimento dos resultados obtidos anteriormente pelas outras avaliações. As pacientes foram instruídas a não revelar o resultado de exames já realizados.

A HSG foi agendada para a primeira fase do ciclo, o mais distante possível do período periovulatório (confirmado por USG prévia imediata ao procedimento). Para execução do exame foi realizada antissepsia da vagina com Povidine, pinçamento do colo uterino com pinça de Pozzi e adaptação da cânula para administração do contraste hidrossolúvel. Foram administradosde 3 a $10 \mathrm{~mL}$ do contraste, sob controle por fluoroscopia, após retirada do espéculo vaginal. Os clichês obrigatórios foram: pelve panorâmica, quatro radiografias que acompanharam a introdução do contraste, tração e elevação do colo uterino, perfil esquerdo e direito e prova de Cotte. Procurou-se avaliar a presença de sinais sugestivos de fatores miometriais (miomas, malformações, passagens linfáticas de contraste, entre outros), endometriais (fa- 
lhas de enchimento, irregularidades do contorno uterino, entre outros) e cervicais (estenoses relativas, acentuada tortuosidade de seu trajeto, divertículos ou insuficiência).

A USG foi realizada com aparelho Aloka SSD2000, com transdutor transvaginal de 5,0 MHz, no mesmo dia da HSG. Foram consideradas as alterações endometriais (espessura, heterogeneidade, áreas hiper ou hipoecogênicas), miometriais (nódulos miomatosos, espessamentos ou irregularidades do halo subendometrial, áreas hiperecogênicas, malformações) e do canal cervical (calcificações e sinais sugestivos de seqüelas inflamatórias).

A HSC foi realizada na primeira fase do ciclo, com histeroscópio rígido de $4,0 \mathrm{~mm}$, após distensão da cavidade uterina com $\mathrm{CO}_{2}$ com controle da pressão intracavitária. Foram avaliados detalhadamente o canal cervical, a cavidade uterina e o aspecto do endométrio.

A RNMP foi realizada com a paciente em semi-repleção vesical entre o $17^{\circ}$ e o $21^{\circ}$ dia do ciclo menstrual. Foram realizados cortes da pelve nos planos sagital, axial e coronal. As imagens foram analisadas nas seqüências $\mathrm{T} 1, \mathrm{~T} 1 \mathrm{com}$ supressão de gordura e T2. O contraste utilizado foi o gadolínio. Foram avaliadas as características do canal endocervical, endométrio, corpo uterino e presença de malformações.

A HSSNG 3D foi realizada na fase folicular do ciclo menstrual seguindo os seguintes passos: colocação de espéculo vaginal, identificação do colo uterino e anti-sepsia da vagina e colo com solução de Povidine a 10\%. Um cateter com balão foi primeiramente lavado e preenchido por solução salina, de forma a não permitir presença de ar no catéter. $O$ cateter foi introduzido no orifício cervical externo até ultrapassar o orifício interno (OCI). O balão foi insuflado com $5 \mathrm{~mL}$ de solução salina e levemente tracionado para ocluir o OCI. A seguir, o espéculo foi removido e foi introduzido transdutor vaginal de 7,5 MHz. Foram injetados de 10 a $30 \mathrm{~mL}$ de solução salina através do cateter enquanto realizava-se exame com aparelho de ultra-sonografia real time de $7,5 \mathrm{MHz}$ e armazenamento de imagens. Foram comparadas todas as alterações encontradas no canal cervical, no relevo e integridade endometriais, na homogeneidade do miométrio (presença de mioma e adenomiose) e a presença de malformações do corpo uterino. A HSG foi considerada padrão-ouro para alterações do canal endocervical, a HSC para alterações endometriais e a RNMP para alterações miometriais.

A análise estatística empregada nas comparações da HSSNG 3D com os demais exames foi o teste kappa. Valores da estatística kappa superiores a $0,75(\kappa>0,75)$ foram considerados indicadores de excelente grau de associação (concordância); entre 0,4 e $0,75(0,4 \leq \kappa \leq 0,75)$, bom grau de associação (concordância) e quando menor que 0,4 $(0 \leq \kappa<0,4)$, grau de associação (concordância) considerado marginal. O valor da estatística kappa está acompanhado da significância calculada (p). Para p menor do que $5 \%(0,05)$, consideramos grau de coincidência significante entre os exames em questão, ou seja, os resultados de um exame assemelham-se, estatisticamente, aos resultados do outro exame.

\section{Resultados}

Das 50 mulheres incluídas no estudo, 12 (24\%) apresentaram alteração em pelo menos um dos exames realizados. Destas, a USG isolada detectou 41,6\% das alterações; a HSG, 75\%, sendo um caso falso-positivo quando comparado com outros exames; a HSSNG 3D, 75\%; a RNMP, 41,6\% e a HSC, 58,3\%. Quando se comparou a HSSNG 3D com o USG, a HSSNG 3D forneceu informações adicionais em 7 casos (58,3\%); comparada com a HSG, obteve informações adicionais em 7 casos (58,3\%); com a HSC, em 4 casos $(32,1 \%)$, e comparada à RNMP, em 6 casos (50\%). Somente em dois casos a HSG detectou alterações do canal endocervical (estreitamento e divertículo) que não foram visualizadas na HSSNG 3D. Em todos os outros casos a HSSNG 3D forneceu o mesmo diagnóstico ou acrescentou informações mais detalhadas em relação aos outros exames realizados; não houve falso-positivos. Foram resumidos na Tabela 1, caso a caso, os resultados comparativos entre cada procedimento realizado pelas mulheres que apresentaram pelo menos um exame alterado. A aplicação da estatística kappa para identificação de possíveis relacionamentos entre os tipos de exame mostrou que a HSSNG 3D apresentou grau de coincidência estatisticamente significante entre as respostas dos exames em questão, ou seja, com o USG (kappa = 0,611; Tabela 2), HSC (kappa = 0,766; Tabela 3) e RNMP (kappa = 0,766; Tabela 4). Comparada com a HSG, a HSSNG 3D também mostrou relação significante de concordância (kappa $=0,593)$, apesar da ocorrência de alterações do canal endocervical (dois casos) não visualizadas na HSSNG 3D (Tabela 5).

Quando associaram-se os resultados da HSG e da HSSNG 3D observou-se que todas as alterações patológicas poderiam ter sido diagnosticadas somente com esses exames. 


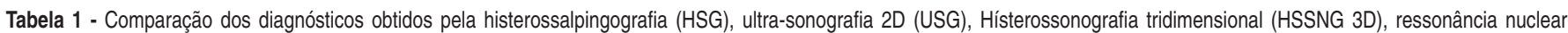
magnética pélvica (RNMP) e histeroscopia diagnóstica (HSC), entre as pacientes inférteis que apresentaram pelo menos um dos exames alterados.

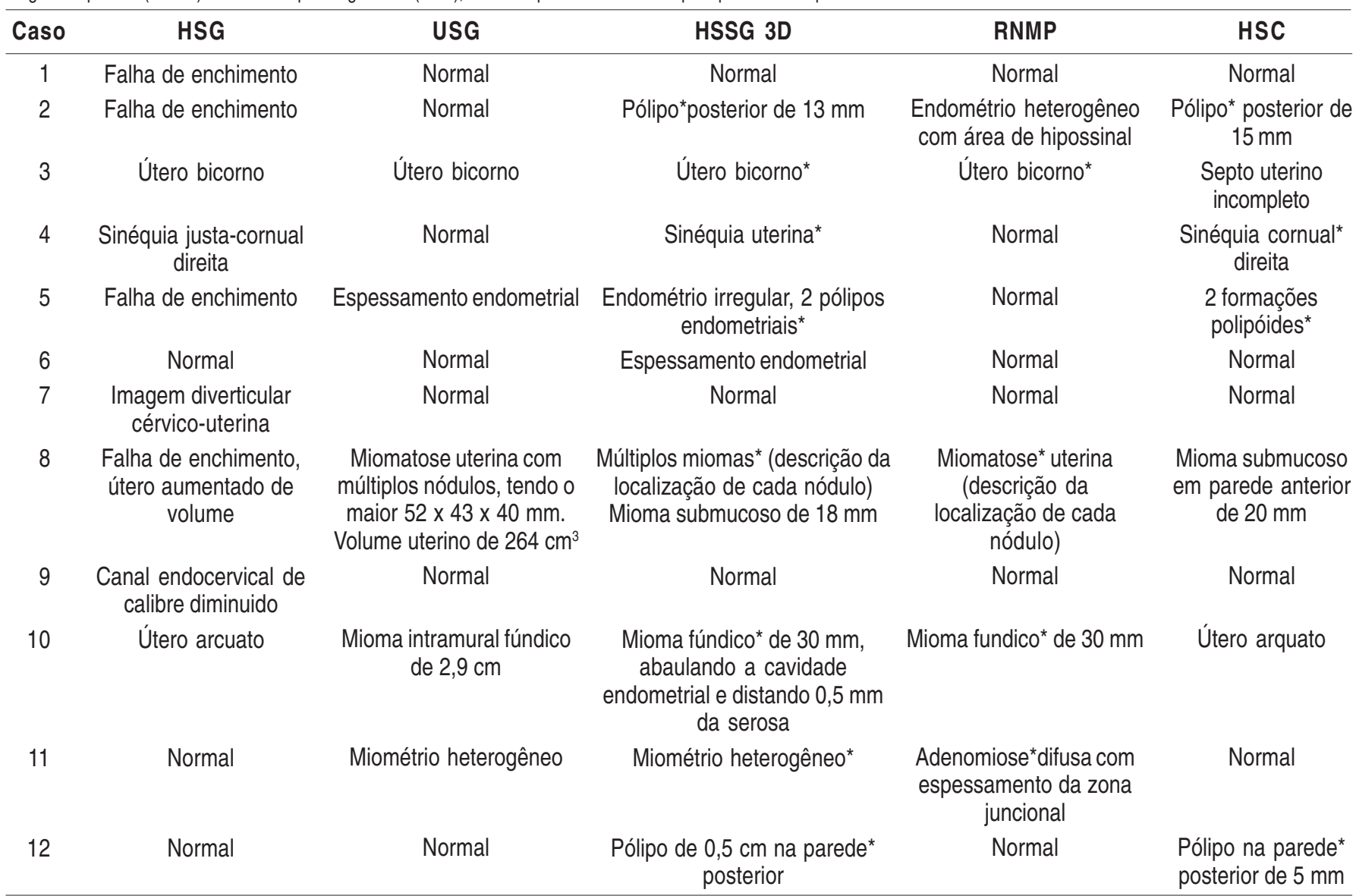

*Diagnósticos concordantes entre HSSNG 3D e o exame considerado padrão-ouro para a alteração.

Tabela 2 - Comparação entre diagnósticos obtidos pela ultra-sonografia (USG) e hísterossonografia 3D (HSSNG 3D) no rastreamento de anomalias uterinas em mulheres inférteis.

\begin{tabular}{lccr}
\hline \multicolumn{4}{c}{ HSSNG 3D } \\
USG & $\mathbf{n}(\%)$ & $\mathbf{n}(\%)$ & Total \\
$\mathbf{N}(\%)$ & Normal & Não-normal & \\
\hline Normal & $41(82,0)$ & $4(8,0)$ & $45(90,0)$ \\
Não-normal & 0 & $5(10,0)$ & $5(10,0)$ \\
Total & $41(82,0)$ & $9(18,0)$ & $50(100,0)$ \\
\hline
\end{tabular}

kappa $=0,672 ; p<0,001$.

Tabela 3 - Comparação entre diagnósticos obtidos pela histeroscopia (HSC) e hísterosonografia 3D (HSSNG 3D) no rastreamento de anomalias uterinas em mulheres inférteis.

\begin{tabular}{|c|c|c|c|}
\hline \multicolumn{4}{|c|}{ HSC } \\
\hline $\begin{array}{l}\text { HSSNG 3D } \\
\text { n (\%) }\end{array}$ & $\begin{array}{c}\mathrm{n}(\%) \\
\text { Normal }\end{array}$ & $\begin{array}{c}n(\%) \\
\text { Não normal }\end{array}$ & Total \\
\hline Normal & $41(82,0)$ & 0 & $41(82,0)$ \\
\hline Não-normal & $3(6,0)$ & $6(12,0)$ & $9(18,0)$ \\
\hline Total & $44(88,0)$ & $6(12,0)$ & $50(100,0)$ \\
\hline
\end{tabular}

kappa $=0,766 ; p<0,001$.
Tabela 4 - Comparação entre diagnósticos obtidos pela da ressonância nuclear magnética pélvica (RNMP) e hísterossonografia 3D (HSSNG 3D) no rastreamento de anomalias uterinas em mulheres inférteis.

\begin{tabular}{lccr}
\hline HSSNG 3D & $\mathbf{n}(\%)$ & $\begin{array}{c}\text { RNMP } \\
\mathbf{n}(\%)\end{array}$ & Total \\
Normal & Normal & Não-normal & \\
Não normal & $41(82,0)$ & 0 & $41(82,0)$ \\
Total & $3(6,0)$ & $6(12,0)$ & $9(18,0)$ \\
\hline
\end{tabular}

kappa $=0,766 ; p<0,001$.

Tabela 5 - Comparação entre diagnósticos obtidos pela histerossalpingografia (HSG) e hísterossonografia 3D (HSSNG 3D) no rastreamento de anomalias uterinas em mulheres inférteis.

\begin{tabular}{lccc}
\hline HSSG 3D & $\mathbf{n}(\%)$ & $\mathbf{n}(\%)$ & Total \\
$\mathbf{n}(\%)$ & Normal & Não normal & \\
\hline Normal & $38(76,0)$ & $3(6,0)$ & $41(82,0)$ \\
Não-normal & $3(6,0)$ & $9(12,0)$ & $9(18,0)$ \\
Total & $41(82,0)$ & $9(18,0)$ & $50(100,0)$ \\
\hline
\end{tabular}

kappa $=0,593 ; p<0,001$. 


\section{Discussão}

Casais com pelo menos dois anos de tentativa de gestação infrutífera são submetidos freqüentemente a extensa rotina de exames que buscam o diagnóstico do fator responsável pela infertilidade. Em aproximadamente 10-15\% dos casos encontra-se um fator uterino isolado ou associado ${ }^{8}$. A HSG e a USG são consideradas provas básicas da pesquisa anatômica ${ }^{9}$.

A HSG apresenta alta sensibilidade e especificidade muito variável quando comparada à HSC $^{10-12}$. A especificidade vai de $15 \%{ }^{11}, 70,1 \%{ }^{10}$, até $81,8 \%^{12}$. Essas variações podem ser explicadas pelos agendamentos no período periovulatório, pela falta de todos os clichês necessários para uma boa avaliação e por um fato importante que é a variabilidade interobservador. Glatstein et al. ${ }^{13}$ mostraram concordância de somente $34 \%$ dos diagnósticos entre diferentes observadores que avaliaram os mesmos clichês. As imagens obtidas pela HSG são indiretas, ou seja, não avaliamos diretamente o relevo endometrial, mas sim as diferentes alterações que este imprime ao contraste que preenche a cavidade. Os resultados falso-positivos quando nos referimos ao fator uterino se relacionam freqüentemente a "falhas de enchimento" provocadas por muco ou até mesmo pregas do endométrio formadas pela pressão exercida pelo contraste, como no primeiro caso (caso 1) apresentado na tabela de resultados. Neste caso todos os outros exames foram normais, sem evidências de imagens intracavitárias ratificando o resultado falso-positivo da HSG.

Os resultados falso-negativos da HSG comumente se apresentam como pólipos, sinéquias e miomas que, completamente envoltos pelo contraste, passam desapercebidos, como nos casos 5 e 12 (pólipo) e no caso 6, no qual a imagem sugestiva de hiperplasia endometrial só foi verificada por meio da HSSNG 3D. Esta permite, como a HSC, a avaliação direta da cavidade, com a vantagem adicional de verificar sua relação com o miométrio, além da problemática referente a "eventuais" baixas taxas de especificidade. Outro fator muito discutido na literatura é a irradiação sobre os ovários durante a prova ${ }^{9,14}$. Ainda assim, é considerada exame obrigatório por $96 \%$ dos especialistas norte-americanos $^{13}$, por melhor avaliar o fator tubo-peritoneal em infertilidade. Além disso, entre todas as provas por nós estudadas, foi a única capaz de predizer as eventuais alterações do canal cervical (casos 7 e 9), de inquestionável importância no que se refere à manutenção das taxas de gravidez em fertilização in vitro.
A simplicidade, o baixo custo e a não invasibilidade tornaram a USG um tempo obrigatório da pesquisa anatômica. Isoladamente, apresenta boa sensibilidade, mas baixa especificidade quando avaliamos a cavidade endometrial. Pólipos e miomas submucosos são freqüentemente descritos à USG como endométrios heterogêneos ou interpretados como espessamentos endometriais (caso 5) ou muitas vezes podem passar desapercebidos se forem de diminutos volumes (casos 2 e 12). Segundo La Torre et al. ${ }^{15}$, a especificidade da USG para o diagnóstico de pólipo é de $69,5 \%$, comparável à da HSC. No que concerne ao miométrio, a sensibilidade e especificidade são elevadas, embora em casos com extensa miomatose uterina (caso 8), a avaliação do número total de nódulos, assim como sua localização, é restrita ${ }^{16}$. Na realidade, muitas das chamadas recidivas de miomas pós-miomectomias são tumorações que já existiam na época da cirurgia e não foram extirpadas pelo fato de o cirurgião desconhecer sua existência e exata localização.

Em nosso serviço, temos grande experiência em RNMP, exame que, apesar do alto custo, evita uma segunda intervenção cirúrgica, por descrever com precisão a disposição dos miomas ${ }^{17,18}$ e por diferenciá-los de adenomiomas ${ }^{7,19}$, que constituiriam contra-indicações relativas para miomectomias laparoscópicas, devido à ausência de planos de clivagem definidos. A RNMP é por nós considerada imprescindível na elaboração da técnica e via de abordagem cirúrgica de doenças miometriais. A RNMP também é considerada método de eleição no diagnóstico de adenomioses incipientes (caso 11) empregando-se como critério o espessamento da zona juncional (área de hipossinal em T2 logo abaixo do endométrio), que na evolução da doença aparece descrita como miométrio heterogêneo ou áreas císticas miometriais à USG. A grande vantagem da USG é a relação funcional que pode ser estabelecida entre as condições endometriais e folículo-ovarianas, assim como a relação uterina com outros órgãos adjacentes, embora a RNMP proporcione visualização ainda mais ampla do compartimento pélvico.

Em casos específicos de malformações uterinas, a HSG tem grande sensibilidade mas há dificuldade para o diagnóstico de úteros que apresentam discreta alteração na morfologia, ou aqueles que apresentam cavidades não comunicantes com o colo uterino, uma vez que não são contras$\operatorname{tadas}^{20}$. Nestas classes de malformações a HSG tem sensibilidade de somente $44,4 \%$.

Outro problema freqüente é a diferenciação entre úteros bicornos e septados (caso 3). Mesmo a associação da HSG, USG e HSC não é suficiente 
para conclusão diagnóstica. A laparoscopia, pela análise direta do fundo uterino, permite a distinção pela verificação da presença de concavidade superior a um centímetro ${ }^{20}$. Muitas vezes, caso não haja suspeita de fator tubo-peritoneal ou endometriose, a laparoscopia pode ser comparada à $\mathrm{RNMP}^{20}$, com a vantagem de a última ser menos invasiva $^{21,22}$.

Considerada por muitos como padrão-ouro na avaliação da cavidade endometrial, a histeroscopia, em muitas situações, não descarta a necessidade de associação com outros exames para diagnósticos mais precisos. Erros diagnósticos podem ser evitados se ocorrer a avaliação anatômica prévia ao exame. Divertículos cervicais podem passar desapercebidos pela HSC, devido à pequena comunicação ou pertuito, sendo melhor diagnosticados pela HSG. A pressão exercida no canal pelo gás ou líquido durante o exame pode levar a modificações que impedem o diagnóstico de estenoses relativas. Pequenas sinéquias nas paredes laterais também podem passar desapercebidas pelo histeroscopista (caso 4), assim como pelo ultra-sonografista. A sensibilidade e o valor preditivo negativo da USG nestes casos são próximos a zero ${ }^{23}$. Entre as desvantagens da HSC estão incluídas: a inadequada visualização da cavidade durante sangramentos uterinos e a impossibilidade de avaliar a porção intramural e serosa assim como os anexos ${ }^{9}$. A partir desta análise, a RNMP pode ser considerada o melhor método para avaliação do miométrio e a HSC o padrão-ouro, apesar de invasiva, para o diagnóstico das alterações da cavidade endometrial, sendo muitas vezes necessária a associação destes dois exames para o diagnóstico definitivo e completo da patologia uterina.

A HSSNG 3D permite, assim como a USG e a RNMP, avaliação miometrial e endometrial adequada e mostra a dinâmica pélvica existente entre órgãos adjacentes, além de associar aspectos anatômicos e funcionais. Soma-se a isso a capacidade da HSSNG 3D, por meio da distensão da cavidade e da avaliação das imagens nos três planos ortogonais, de reconstruir a cavidade endometrial com imagens semelhantes às obtidas pela HSC, conforme verificado nos poucos trabalhos existentes na literatura ${ }^{16,24}$ e, segundo pudemos avaliar, neste estudo, apesar da pequena casuística apresentada.

Uma das vantagens da técnica é permitir a avaliação dos dados volumétricos arquivados no aparelho que permitem rotações, manipulações, zoom e obtenção de diferentes cortes para melhor detalhamento da doença sem a necessidade do tempo real. A tecnologia 3D permite obtenção de corte coronal da cavidade uterina, podendo-se observar a relação entre endométrio e miométrio da região fúndica produzindo boas imagens das regiões cornuais, sempre problemáticas com o uso de USG. O tamanho e a localização da base de implantação são informados com precisão ${ }^{15}$ (casos 2, 5 e 12). É possivel imaginar que a injeção de líquidos e os diferentes cortes e composições de imagens obtidas pelos aparelhos tridimensionais possam contornar algumas dificuldades das HSC, principalmente quanto à penetração do histeroscópio (sem anestesia) ou delimitação de pedículos de grandes tumorações pela dopplerfluxometria. A especificidade da HSSNG 3D no diagnóstico de pólipos endometriais é de 100\% comparado aos resultados anatomopatológicos.

Bonilla-Musoles et al. ${ }^{3}$ estudaram 36 pacientes com sangramento pós-menopausa submetidas a HSSNG 3D e foi encontrada correlação de 100\% entre este exame e os achados histeroscópicos, incluindo diagnóstico de pólipo e imagem sugestiva de hiperplasia focal. Em estudo de rastreamento de anomalias uterinas em 267 mulheres inférteis ${ }^{25}, 108$ apresentaram alterações endometriais ou miometriais. Todas foram submetidas previamente a HSSNG 3D e, em seguida, à HSC cirúrgica. Todos os casos com pólipo endometrial, mioma submucoso, útero arquato e septo uterino suspeitos na HSSNG 3D foram confirmados na HSC. Somente um de quatro casos de sinéquia uterina foi diagnosticado no pré-operatório. LevToaff et al. ${ }^{16}$ mostraram que a HSSNG 3D promoveu informações diagnósticas adicionais em $92 \%$ dos casos quando comparou este exame à HSG.

Acreditamos que a HSSNG 3D, concomitante com a HSG, será suficiente para o diagnóstico cérvico-uterino em infertilidade. Apesar do custo atual dos aparelhos 3D serem proibitivos, futuramente a complementação com a RNMP será restrita, assim como a realização de HSC diagnósticas não será obrigatória, podendo-se partir diretamente para a histeroscopia cirúrgica nos casos em que houver necessidade ${ }^{15,23}$. Dessa forma, poderemos reduzir o número de exames realizados por cada paciente na elucidação de doenças uterinas, diminuindo os custos totais finais, a ansiedade e, conseqüentemente, o retardo no tratamento.

\section{ABSTRACT}

Purpose: to compare the information obtained with pelvic and transvaginal ultrasonography (USG), hysterosalpingography (HSG), diagnostic hysteroscopy (HSC), pelvic nuclear magnetic resonance imaging (PNMR), three- 
dimensional hysterosonography (3D HSSNG), to optimize and simplify the investigation about cervical and corporeal uterine factors in conjugal infertility.

Methods: in the period between January and July 2003, fifty women reporting infertility for at least two years were submitted to USG, HSG, HSC, PNMR, and 3D HSSNG as tracking examinations for uterine factor diagnosis. The endocervical canal, as well as the endometrium, myometrium, and the presence of uterine malformations were investigated. The results of each examination were analyzed and compared.

Results: of the 50 women studied, 12 (24\%) presented alteration in at least one of the examinations. When $3 D$ HSSNG was compared to USG, 3D HSSNG provided additional information in 7 cases (58.3\%); when compared to HSG, it provided additional information in 7 cases (58.3\%); when compared to $\mathrm{HSC}$, it provided additional information in 4 cases $(32.1 \%)$, and when compared to PNMR, it provided additional information in 6 cases (50\%). There were only two cases in which HSG detected alterations of the endocervical canal that were not visualized using $3 D$ HSSNG. In the other cases 3D HSSNG imparted the same diagnosis; furthermore, it provided additional information in comparison to the other examinations. Statistical analysis using the kappa test demonstrated that the diagnoses obtained by 3D HSSNG were in agreement with those obtained with USG, HSG and PNMR $(p<0,05)$. When the HSG and $3 D$ HSSNG results were combined, all conditions associated with infertility could be precisely diagnosed, using only these examinations.

Conclusion: the association of the HSG with 3D HSSNG may be sufficient for the diagnosis of cervical and corporeal uterine factors in infertility, reducing the number of examinations for each patient, the total cost, as well as the anxiety and the delay in treatment.

KEYWORDS: Infertility. Hysterosalpingography. Nuclear magnetic resonance. Histeroscopy.

\section{Agradecimento}

Agradecemos ao Núcleo de Apoio à Publicação da Faculdade de Ciências Médicas da Santa Casa de São Paulo (NAP-SC) o suporte técnico-científico à publicação deste manuscrito.

\section{Referências}

1. Hamilton JA, Larson AJ, Lower AM, Hasnain S, Grudzinskas JG. Routine use of saline hysterosonography in 500 consecutive, unselected, infertile women. Hum Reprod 1998; 13:2463-73.

2. Fedele L, Parazzini F, Luchini L, Mezzopane R, Tozzi L, Villa L. Recurrence of fibroids after myomectomy: a transvaginal ultrasonographic study. Hum Reprod 1995; 10:1795-6.
3. Bonilla-Musoles F, Raga F, Osborne NG, Blanes J, Coelho F. Three-dimensional hysterosonography for the study of endometrial tumors: comparison with conventional transvaginal sonography, hysterosalpingography, and hysteroscopy. Gynecol Oncol 1997; 65:245-52.

4. Siegler AM. Hysterography and hysteroscopy in the infertile patient. J Reprod Med 1977; 18:143-8.

5. Valle RF. Hysteroscopy in the evaluation of female infertility. Am J Obstet Gynecol 1980; 137:425-31.

6. Richman TS, Viscomi GN, deCherney A, Polan ML, Alcebo LO. Fallopian tubal patency assessed by ultrasound following fluid injection. Work in progress. Radiology 1984; 152:507-10.

7. Ascher SM, Arnold LL, Patt RH, et al. Adenomyosis: prospective comparison of MR imaging and transvaginal sonography. Radiology 1994; 190:803-6.

8. Wallach EE. The uterine factor in infertility. Fertil Steril 1972; 23:138-58.

9. Brown SE, Coddington CC, Schnorr J, Toner JP, Gibbons W, Oehninger S. Evaluation of outpatient hysteroscopy, saline infusion hysterosonography, and hysterosalpingography in infertile women: a prospective, randomized study. Fertil Steril 2000; 74:1029-34.

10.Wang CW, Lee CL, Lai YM, Tsai CC, Chang MY, Soong YK. Comparison of hysterosalpingography and hysteroscopy in female infertility. J Am Assoc Gynecol Laparosc 1996; 3:581-4.

11.Golan A, Eilat E, Ron-El R, Herman A, Soffer Y, Bukovsky I. Hysteroscopy is superior to hysterosalpingography in infertility investigation. Acta Obstet Gynecol Scand 1996; 75:654-6.

12.Gaglione R, Valentini AL, Pistilli E, Nuzzi NP. A comparison of hysteroscopy and hysterosalpingography. Int J Gynaecol Obstet 1996; 52:151-3.

13.Glatstein IZ, Sleeper LA, Lavy Y, et al. Observer variability in the diagnosis and management of the hysterosalpingogram. Fertil Steril 1997; 67:233-7.

14.Hauge K, Flo K, Riedhart M, Granberg S. Can ultrasound-based investigations replace laparoscopy and hysteroscopy in infertility? Eur J Obstet Gynecol Reprod Biol 2000; 92:167-70.

15.La Torre R, De Felice C, De Angelis C, Coacci F, Mastrone M, Cosmi EV. Transvaginal sonographic evaluation of endometrial polyps: a comparison with two dimensional and three dimensional contrast sonography. Clin Exp Obstet Gynecol 1999; 26:171-3.

16.Lev-Toaff AS, Pinheiro LW, Bega G, Kurtz AB, Goldberg BB. Three-dimensional multiplanar sonohysterography: comparison with conventional two-dimensional sonohysterography and X-ray hysterosalpingography. J Ultrasound Med 2001; 20:295-306. 
17.Dudiak CM, Turner DA, Patel SK, Archie JT, Silver B, Norusis M. Uterine leiomyomas in the infertile patient: preoperative localization with MR imaging versus US and hysterosalpingography. Radiology 1988; 167:627-30.

18.Zawin M, McCarthy S, Scoutt LM, Comite F. Highfield MRI and US evaluation of the pelvis in women with leiomyomas. Magn Reson Imaging 1990; 8:371-6.

19.Arnold LL, Ascher SM, Schruefer JJ, Simon JA. The nonsurgical diagnosis of adenomyosis. Obstet Gynecol 1995; 86:461-5.

20.Kinkel K, Vincent B, Balleyguier C, Helenon O, Moreau J. Value of MR imaging in the diagnosis of benign uterine conditions. J Radiol 2000; 81:773-9.

21.Fedele L, Dorta M, Brioschi D, Massari C, Candiani GB. Magnetic resonance evaluation of double uteri. Obstet Gynecol 1989; 74:844-7.
22.Woodward PJ, Wagner BJ, Farley TE. MR imaging in the evaluation of female infertility. Radiographics 1993; 13:293-310.

23.Soares SR, Barbosa dos Reis MM, Camargos AF. Diagnostic accuracy of sonohysterography, transvaginal sonography, and hysterosalpingography in patients with uterine cavity diseases. Fertil Steril 2000; 73:406-11.

24.Balen FG, Allen CM, Gardener JE, Siddle NC, Lees WR. 3-dimensional reconstruction of ultrasound images of the uterine cavity. Br J Radiol 1993; 66:588-91.

25.Radoncic E, Funduk-Kurjak B. Three-dimensional ultrasound for routine check-up in vitro fertilization patients. Croat Med J 2000; 41:262-5.

Recebido em: 23/8/2004 Aceito com modificações em: 8/11/2004 